\title{
Effect of Herbicidal Weed Control on Growth and Yield of Soybean
}

\author{
Bharat Lal Meena ${ }^{1 *}$, D.S. Meena ${ }^{2}$, Baldev Ram², M.K. Sharma ${ }^{3}$, \\ Chirag Gautam ${ }^{4}$ and Gajendra Nagar ${ }^{1}$
}

${ }^{1}$ Agronomy, College of Agriculture, Kota- 324001, India

${ }^{2}$ Agronomy, Agricultural Research Station (AU), Kota - 324001, India

${ }^{3}$ Soil Science \& Ag. Chemistry, Agricultural Research Station (AU), Kota - 324001, India

${ }^{4}$ Plant Pathology, Agricultural Research Station (AU), Kota - 324001, India

*Corresponding author

\section{A B S T R A C T}

\section{Keywords}

Soybean, Weed, Weed control efficiency

\section{Article Info}

Accepted:

20 September 2020

Available Online:

10 October 2020
A field experiment was conducted during kharif, 2019 at Agricultural Research Station, Ummedganj, Kota. Dominating weed flora of the experimental field consisted of Cynodon dactylon L., Eleusine indica L., Echinochloacrusgalliand Echinochloa colona among grassy weeds, Boerhaavia diffusa L., Convolvulus arvensis L., Commelina benghalensis L., Digera arvensis Forsk., Celosia argentea L. among broad leaved weedsand Cyperus rotundus L. in the sedge category of weeds. The results showed that all weed control treatments recorded significantly higher values of growth parameters plant height, branches plant ${ }^{-1}$, dry matter accumulation and yield attributes viz. pods plant ${ }^{-1}$, seeds $\operatorname{pod}^{-1}$, seed yield plant $^{-1}$ and seed index as compared to weedy check. Two hand weeding at 20 and 40 DAS recorded significantly lower weed density, weed dry weight, maximum weed control efficiency and significantly improved the growth characters, yield and yield attributing characters followed by post emergence application of sodium acifluorfen $16.5 \%$ + clodinafop propargyl 8\% (premix) @ $165+80 \mathrm{~g}$ a.i. ha ${ }^{-1}$ at 20 DAS.

\section{Introduction}

Soybean (Glycine max L. Merrill) is an important oilseed and food grain legume crop. Being a rainy season crop soybean faces severe weed competition during early stages of crop growth, resulting in a loss of potential yield, depending on the weed intensity, nature, environmental condition and duration of weed competition. The competitional stress of weeds on crop for nutrients, water, light and space are responsible for poor yield of soybean. Weeds can cause significant seed yield losses in soybean (Jha et al. 2014). If weeds are not controlled during critical periods of crop-weed competition, there is identical reduction in the yields of soybean up-to $58-85 \%$, depending upon the types and 
intensity of weeds. Thus, it is important to keep the crop free from weeds as far as possible so as to get higher yield from soybean (Kewat et al., 2000). Manual weeding is the best option for weed control, but it is costly, time consuming and difficult due to intermittent rains. Timely unavailability of adequate labourers also possesses serious problem. Therefore, it is necessary, to find out the alternative methods for reducing the weed load during early growth period of soybean to get economical yields. Thus, herbicidal weed control remains only the choice under such situation to minimize the weed menace economically. Therefore, keeping the above facts in view, the present experiment has been carried outto find out effective weed control method.

\section{Materials and Methods}

The experiment was conducted during kharif season of 2019 at Agricultural Research Station, Ummedganj, Kota, which is situated at $25^{\circ} 21^{\prime} \mathrm{N}$ latitude and $75^{\circ} 87^{\prime} \mathrm{E}$ longitude at an altitude of $271 \mathrm{~m}$ above mean sea level. It falls under agroclimatic zone V (Humid South Eastern Plain) of Rajasthan and agroclimatic zone VIII (Central Plateau and Hills) of India. The experiment was laid out in RBD with eight treatments, replicated three times. The experiment comprises eight treatments viz. pendimethalin $30 \% \mathrm{EC} @ 1.0 \mathrm{~kg}$ a.i.ha ${ }^{-1}$ as PE, pendimethalin 30\% EC + imazethapyr 2\% SL (premix) @ $960 \mathrm{~g}$ a.i. ha ${ }^{-1}$ as PE, sodium acifluorfen $16.5 \%$ + clodinafop propargyl 8\% EC (premix)@ $165+80 \mathrm{~g}$ a.i. ha ${ }^{-1}$ at 20 DAS, quizalofop-ethyl 5\% EC @ $50 \mathrm{~g}$ a.i. ha ${ }^{1}$ at 20 DAS, imazethapyr 10\% SL @ $100 \mathrm{~g}$ a.i. ha $^{-1}$ at $20 \mathrm{DAS}$, imazethapyr $3.75 \%+$ propaquizafop 2.5\% ME (premix) @ 50+75 $\mathrm{g}$ a.i. ha $\mathrm{h}^{-1}$ at $20 \mathrm{DAS}$, two hand weeding at 20 \& 40 DAS and weedy check.

The soil of the experimental field was clay loam in texture and the soil having medium fertility status. Soybean variety RKS-113 (Kota Soya-1) was used as experimental material developed at ARS, Kota (Rajasthan).

\section{Results and Discussion}

Dominating weed flora of the experimental field consisted of Cynodon dactylon (L.), Digitaria arvensis Forsk, Eleusine indica L. and Echinochloa colonum among grassy weeds, Boerhavia diffusa L., Convolvulus arvensis L., Commelina bengalensis L., Celosia argentea L. among broad leaved weedsand Cyperus rotundus L. in the sedge category of weeds. Category wise weed data recorded in weedy check plots indicated that there was pre-dominance of grassy weeds $(48.60 \%)$ as compared to broad leaved weeds $(39.49 \%)$ and sedges weeds $(11.91 \%)$ in the experimental field. Almost similar results were observed by Meena et al. (2011).

The effect of various weed control treatments and total weeds population was found significantly reduced at all stages. Lowest weed density and dry weight was recorded under two hand weeding, which was superior as compared to all the herbicidal treatments in the agreement results reported by Meena et al., (2009). Among herbicidal weed control treatments post emergence application of sodium acifluorfen $16.5 \%+$ clodinafop propargyl 8\% EC (premix) @ 165+80 g a.i. $\mathrm{ha}^{-1}$ at 20 DAS found most effective in reducing significantly weed density and weed dry weight, which was superior as compared to over rest of herbicidal treatments. Similar results were observed by Verma and Kushwaha, 2019. The highest weed control efficiency at 60 DAS was recorded in treatment hand weeding twice at 20 and 40 DAS (77.79\%), followed by application of sodium acifluorfen $16.5 \%+$ clodinafop propargyl 8\% EC (premix) @165+80 g a.i. ha $^{-1}$ at 20 DAS (66.67\%) (Table 1 and 2). 
Table.1 Effect of herbicidal weed control on yield attributing characters and yield of soybean

\begin{tabular}{|c|c|c|c|c|c|c|}
\hline Treatments & $\begin{array}{l}\text { Pods } \\
\text { plant }^{-1} \\
(\text { No. })\end{array}$ & $\begin{array}{l}\text { Seeds } \\
\operatorname{pod}^{-1} \\
\text { (No.) }\end{array}$ & $\begin{array}{c}\text { Seed } \\
\text { yield } \\
\text { plant }^{-1}(g)\end{array}$ & $\begin{array}{c}\text { Seed } \\
\text { index } \\
(\mathrm{g})\end{array}$ & $\begin{array}{c}\text { Seed } \\
\text { yield } \\
\left(\mathrm{kg} \mathrm{ha}^{-1}\right)\end{array}$ & $\begin{array}{c}\text { Straw } \\
\text { yield } \\
\left(\mathrm{kg} \mathrm{ha}^{-1}\right)\end{array}$ \\
\hline $\begin{array}{l}\text { Pendimethalin } 30 \% \text { EC @1.0 kg a.i. ha }{ }^{-1} \text { as } \\
\text { PE }\end{array}$ & 33.9 & 2.00 & 4.29 & 11.00 & 1225 & 1792 \\
\hline $\begin{array}{l}\text { Pendimethalin } 30 \% \text { EC + imazethapyr } 2 \% \\
\text { SL (premix)@960 g a.i. ha }{ }^{-1} \text { as PE }\end{array}$ & 37.2 & 2.13 & 4.85 & 11.20 & 1475 & 2128 \\
\hline $\begin{array}{l}\text { Sodium acifluorfen } 16.5 \%+\text { clodinafop } \\
\text { propargyl } 8 \% \text { EC (premix) @ } 165+80 \text { g a.i. } \\
\text { ha }^{-1} \text { at } 20 \text { DAS }\end{array}$ & 41.2 & 2.20 & 5.32 & 11.33 & 1550 & 2233 \\
\hline $\begin{array}{l}\text { Quizalofop ethyl 5\% EC @ } 50 \mathrm{~g} \text { a.i. ha }{ }^{-1} \text { at } 20 \\
\text { DAS }\end{array}$ & 34.9 & 2.13 & 4.56 & 10.97 & 1325 & 1930 \\
\hline $\begin{array}{l}\text { Imazethapyr } 10 \% \text { SL } 100 \text { g a.i. ha } \text { ha }^{-1} \text { at } \\
\text { DAS }\end{array}$ & 35.9 & 2.13 & 4.65 & 11.07 & 1425 & 2091 \\
\hline $\begin{array}{l}\text { Imazethapyr } 3.75 \% \text { + propaquizafop } 2.5 \% \\
\text { ME (premix) } 50+75 \text { g a.i. ha }{ }^{-1} \text { at } 20 \text { DAS }\end{array}$ & 39.5 & 2.13 & 5.21 & 11.10 & 1520 & 2190 \\
\hline Hand weeding at $20 \& 40$ DAS & 46.7 & 2.27 & 6.10 & 11.43 & 1800 & 2592 \\
\hline Weedy check & 24.1 & 1.93 & 2.82 & 10.93 & 700 & 1028 \\
\hline SEm \pm & 1.27 & 0.09 & 0.17 & 0.20 & 40.53 & 63.72 \\
\hline CD at $5 \%$ & 3.86 & NS & 0.51 & NS & 122.93 & 193.28 \\
\hline $\mathrm{CV}$ & 6.01 & 7.43 & 6.12 & 3.08 & 5.10 & 5.52 \\
\hline
\end{tabular}


Table.2 Effect of herbicide weed control on density $\mathrm{m}^{-2}$ of weeds and weed control efficiency at 60 DAS

\begin{tabular}{|c|c|c|c|c|c|}
\hline Treatments & $\begin{array}{l}\text { Grassy weeds } \\
\text { (dry weight } \mathrm{g} \\
\left.\mathrm{m}^{-2}\right)\end{array}$ & $\begin{array}{l}\text { Broad leaved weeds } \\
\left(\text { dry weight } \mathrm{g} \mathrm{m}^{-2} \text { ) }\right.\end{array}$ & $\begin{array}{c}\text { Sedges } \\
(\text { dry weight } g \\
\left.\mathrm{m}^{-2}\right)\end{array}$ & $\begin{array}{l}\text { Total weeds } \\
\left(\text { dry weight } \mathrm{g} \mathrm{m}^{-}\right. \\
\mathbf{2} \text { ) }\end{array}$ & $\begin{array}{l}\text { Weed Control } \\
\text { Efficiency }(\%)\end{array}$ \\
\hline & 60 DAS & 60 DAS & 60 DAS & 60 DAS & 60 DAS \\
\hline $\begin{array}{l}\text { Pendimethalin } 30 \% \text { EC @1.0 kg a.i. } \\
\text { ha }^{-1} \text { as PE }\end{array}$ & $\begin{array}{c}5.05 \\
(24.50)\end{array}$ & $\begin{array}{c}4.59 \\
(20.17)\end{array}$ & $\begin{array}{c}2.38 \\
(4.68)\end{array}$ & $\begin{array}{c}7.09 \\
(49.34)\end{array}$ & 44.39 \\
\hline $\begin{array}{l}\text { Pendimethalin 30\% EC + } \\
\text { imazethapyr 2\% SL (premix)@960 g } \\
\text { a.i. ha }{ }^{-1} \text { as PE }\end{array}$ & $\begin{array}{c}4.09 \\
(15.77)\end{array}$ & $\begin{array}{c}3.76 \\
(13.13)\end{array}$ & $\begin{array}{c}2.18 \\
(3.74)\end{array}$ & $\begin{array}{c}5.80 \\
(32.63)\end{array}$ & 63.28 \\
\hline $\begin{array}{l}\text { Sodium acifluorfen } 16.5 \%+ \\
\text { clodinafop propargyl } 8 \% \mathrm{EC} \\
\text { (premix)@165+80 g a.i. ha }{ }^{-1} \text { at } 20 \\
\text { DAS }\end{array}$ & $\begin{array}{c}4.01 \\
(15.07)\end{array}$ & $\begin{array}{c}3.52 \\
(11.38)\end{array}$ & $\begin{array}{c}2.04 \\
(3.17)\end{array}$ & $\begin{array}{c}5.53 \\
(29.62)\end{array}$ & 66.67 \\
\hline $\begin{array}{l}\text { Quizalofop ethyl 5\% EC @ } 50 \mathrm{~g} a . i . \\
\text { ha }^{-1} \text { at } 20 \text { DAS }\end{array}$ & $\begin{array}{c}3.76 \\
(13.17)\end{array}$ & $\begin{array}{c}4.81 \\
(22.12)\end{array}$ & $\begin{array}{c}2.24 \\
(4.03)\end{array}$ & $\begin{array}{c}6.35 \\
(39.32)\end{array}$ & 55.71 \\
\hline $\begin{array}{l}\text { Imazethapyr } 10 \% \text { SL } 100 \mathrm{~g}_{\text {a.i. }} \mathrm{ha}^{-1} \\
\text { at } 20 \text { DAS }\end{array}$ & $\begin{array}{c}4.48 \\
(19.08)\end{array}$ & $\begin{array}{c}3.97 \\
(14.80)\end{array}$ & $\begin{array}{c}2.21 \\
(3.87)\end{array}$ & $\begin{array}{c}6.22 \\
(37.75)\end{array}$ & 57.52 \\
\hline $\begin{array}{l}\text { Imazethapyr } 3.75 \%+\text { propaquizafop } \\
2.5 \% \text { ME (premix) } 50+75 \mathrm{~g}^{\text {a.i. }} \text { ha }^{-1} \text { at } \\
20 \text { DAS }\end{array}$ & $\begin{array}{c}4.04 \\
(15.33)\end{array}$ & $\begin{array}{c}3.67 \\
(12.51)\end{array}$ & $\begin{array}{c}2.18 \\
(3.77)\end{array}$ & $\begin{array}{c}5.71 \\
(31.61)\end{array}$ & 64.39 \\
\hline Hand weeding at $20 \& 40$ DAS & $\begin{array}{c}3.33 \\
(10.23)\end{array}$ & $\begin{array}{c}2.87 \\
(7.23)\end{array}$ & $\begin{array}{c}1.81 \\
(2.28)\end{array}$ & $\begin{array}{c}4.55 \\
(19.74)\end{array}$ & 77.79 \\
\hline Weedy check & $\begin{array}{c}6.87 \\
(46.33)\end{array}$ & $\begin{array}{c}6.25 \\
(38.27)\end{array}$ & $\begin{array}{c}2.74 \\
(6.56)\end{array}$ & $\begin{array}{c}9.48 \\
(88.83)\end{array}$ & 0.00 \\
\hline $\mathrm{SEm} \pm$ & 0.17 & 0.16 & 0.07 & 0.10 & 1.33 \\
\hline CD at $5 \%$ & 0.51 & 0.49 & 0.22 & 0.29 & 4.03 \\
\hline $\mathbf{C V}$ & 6.59 & 6.75 & 5.54 & 2.61 & 4.28 \\
\hline
\end{tabular}

Note: Data in parenthesis are original values of weed density and dry weight. Square root transformed value $(\sqrt{ } \mathrm{x}+1)$ of weed density used for statistical analysis 
All the weed control treatments gave significantly more plant height and higher branches plant ${ }^{-1}$ than weedy check. Hand weeding twice at 20 and 40 DAS was recorded taller plants and maximum branches plant $^{-1}$ followed by application of sodium acifluorfen $16.5 \%$ + clodinafop propargyl 8\% EC (premix) @ $165+80 \mathrm{~g}$ a.i. ha $^{-1}$ at 20 DAS. The increased dry matter accumulation by crop in plots receiving hand weeding twice at 20 and 40 DAS followed by application of sodium acifluorfen $16.5 \%$ + clodinafop propargyl 8\% EC (premix) @ 165+80 g a.i. ha ${ }^{-1}$ at 20 DAS, which was at par with application of imazethapyr $3.75 \%+$ propaquizafop $2.5 \% \mathrm{ME}$ (premix) $50+75 \mathrm{~g}$ a.i. $\mathrm{ha}^{-1}$ at 20 DAS. Hand weeding twice at 20 and 40 DAS recorded higher values of yield attributing characters like pods plant ${ }^{-1}$, seeds $\operatorname{pod}^{-1}$, seed index and seed yield plant ${ }^{-1}$ then other treatments except application of sodium acifluorfen $16.5 \%$ + clodinafop propargyl 8\% EC (premix) @ 165+80 g a.i. ha ${ }^{-1}$ at 20 DAS, which was closely followed to hand weeding. The seed and straw yield was recorded significantly higher under two hand weeding (1800 \& $2592 \mathrm{~kg} \mathrm{ha}^{-1}$, respectively)followed by post emergence application of sodium acifluorfen $16.5 \%$ + clodinafop propargyl 8\% (premix) @ $165+80 \mathrm{~g}$ a.i. ha ${ }^{-1}$ at 20 DAS (1550 \& $2233 \mathrm{~kg} \mathrm{ha}^{-1}$, respectively). Manjunath and Hosmath (2016) and Kamble et al., (2017) also reported that hand weeding as an effective method of weed control for achieving the maximum yield of soybean. Harithavardhini et al., 2016 and Verma andKushwaha, 2019 was reported that sodium acifluorfen $16.5 \%+$ clodinafop propargyl 8\% EC (premix) @187.5 $\mathrm{g}$ a.i. ha ${ }^{-1}$ gave higher yield among herbicidal weed control treatments.

\section{References}

Harithavardhini J, Jayalalitha K, Ashoka Rani Y and Krishnaveni B, 2016. Efficacy of post emergence herbicides on weed control efficiency, partitioning of dry matter and yield of blackgram [Vigna mungo (L.) Hepper]. International Journal of Food, Agriculture and Veterinary Sciences, 6(2): 39-44.

Jha BK, Chandra R and Singh R, 2014. Influence of post emergence herbicides on weeds, nodulation and yields of soybean and soil properties. Legume Research, 37(1): 47-54.

Kamble AB, Nagre BS and Dhonde MB. 2017. Crop geometry and weed management effect on weed dynamics in soybean. (In) Proceedings of Biennial Conference on “Doubling Farmers' Income by 2022: The Role of Weed Science", 1-3 March, 2017, Udaipur. Indian Society of Weed Science, Jabalpur, India pp: 127.

Kewat ML, Pandey J, Yaduraju NT and Kulshreshtha G. 2000. Economic and ecofriendly weed management in soybean. Indian Journal of Weed Science32(3\&4): 135-139.

Manjunath NC and Hosmath JA. 2016. Effect of sequential application of herbicides on weed dynamics and yield of soybean. Journal of Farm Science 29(2): 187-189.

Meena DS, Ram Baldev and Jadon C, 2009. Effect of integrated weed management on growth and productivity of soybean. Indian Journal of Weed Science 41(1\&2): 93-95.

Meena DS, Ram Baldev, Jadon Chaman and Tetarwal JP. 2011. Efficacy of imazethapyr on weed management in soybean. Indian Journal of Weed Science, 43(3\&4): 169171.

Verma L and Kushwaha HS, 2019.Evaluation of different herbicides against weeds in mungbean (Vigna radiata L.). Legume Research, 4048: 1-6.

\section{How to cite this article:}

Bharat Lal Meena, D.S. Meena, Baldev Ram, M.K. Sharma, Chirag Gautam and Gajendra Nagar. 2020. Effect of Herbicidal Weed Control on Growth and Yield of Soybean. Int.J.Curr.Microbiol.App.Sci. 9(10): 2880-2884. doi: https://doi.org/10.20546/ijcmas.2020.910.347 